\title{
Pseudopotential theory of Auger processes in CdSe quantum dots
}

\author{
Lin-Wang Wang \\ NERSC, Lawrence Berkeley National Laboratory, Berkeley, CA, 94720 \\ Marco Califano, Alex Zunger \\ National Renewable Energy Laboratory, Golden, CO, 80401 \\ Alberto Franceschetti \\ Oak Ridge National Laboratory, Oak Ridge, TN 37831
}

\begin{abstract}
Auger rates are calculated for CdSe colloidal quantum dots using atomistic pseudopotential wavefunctions. We predict the dependence of Auger electron cooling on size and on correlation effects (included via configuration interaction). Auger multi-exciton recombination rates are predicted for bi-excitons as well as for tri-excitons. The results agree with recent measurements and shed light on the significance of the dot surface on Auger multi-exciton decay.

PACS numbers: 71.15.-m, 71.55.-i
\end{abstract}

Auger effects are expected to play a central role in carrier relaxation in nanostructures [1]-[10]. Two types of Auger effects, ilustrated in Fig. 1, are prominent:

(i) Electron cooling (Figs. 1a,b: In the 3D bulk, or a $2 \mathrm{D}$ quantum well, the relaxation of an excited electron to its ground state usually occurs by phonon emission. The discrete nature of the electronic states of $0 \mathrm{D}$ dots is expected [2] to prevent phonon-assisted electron relaxation (phonon bottleneck). Multi-phonon (combined LO \pm LA) effects [3], or polaronic effects $[4,5]$, may allow electronic relaxation to occur within a limited energy range around the phonon energy $\hbar \omega_{0}$. However, this may not be sufficient to remove the phonon bottleneck in small, strongly confined quantum dots. On the other hand, electron relaxation rates in CdSe quantum dots were observed to be fast $(\tau \sim 0.3 \mathrm{ps}$ in $2.3 \mathrm{~nm}$ radius nanocrystals [6], $\tau=0.9-1.2 \mathrm{ps}$ for samples of size $4.3 \mathrm{~nm}$ [7]). It was proposed [8] that in a photoexcited quantum dot the "hot" electron can transfer its energy to the hole via an Auger process involving electron-hole scattering (Fig. 1a,b).

(ii) Auger multi-exciton recombination (Fig. 1c,d): A ground-state biexciton can decay into an excited-state monoexciton. Due to a large number of final monoexciton excited states, the efficiency of this process competes with radiative recombination (i.e. $\tau_{e-h}^{2 \rightarrow 1}<\tau_{\text {rad }}$ ), and effectively quenches the PL intensity [9]. These events can lead to photoionization (when one of the final electron or hole states is unbound), which was invoked to explain PL intermittency effects [10]. The inverse Auger process (creation of two e-h pairs from a single excited exciton) has been advocated [11] as a mechanism of enhancing solar cell efficiency. A similar Auger process involves the decay of a ground state tri-exciton into an excited bi-exciton; this $3 \rightarrow 2$ Auger decay (Fig. 1d) can be even faster than the $2 \rightarrow 1$ decay (Fig. 1c). Yet another type of Auger process involves the decay of a ground state trion into a hot electron $\left(\tau_{e}\right.$ in Fig. 1e) or a hot hole $\left(\tau_{h}\right.$ in Fig. 1f).

All Auger effects illustrated in Fig. 1 are at the heart of quantum dot carrier dynamics, and produce phenomena distinct from bulk physics. Recently, femtosecond carrier dynamics studies (see $[1,6,9]$ and references therein) have been performed on colloidal quantum dots. These experiments have revealed various carrier relaxation times, which have been attributed to different Auger relaxation processes. Since, however, the interpretation of the results in terms of specific decay mechanisms is uncertain, there is a need for accurate theoretical calculations for specific Auger decay processes. Auger effects in quantum dots have been previously modeled using $\mathbf{k} \cdot \mathbf{p}$ $[8,13]$ and tight-binding [14] Hamiltonians. However, the conventional $\mathbf{k} \cdot \mathbf{p}$ description for the highly excited states involved in Auger transitions might not be sufficiently accurate [15]. Furthermore, the choice of the boundary conditions for the $\mathbf{k} \cdot \mathbf{p}$ wavefunctions near the surface could be problematic [16], and as we will see later, such region plays a critical role in some of the Auger processes. There are also problems due to the lack of atomistic description of the wavefunctions for multi-exciton Auger effects which involve exchange- and Coulomb-like integrals. The same problems exist in the tight-binding method which lack explicit basis functions [14]. Thus, there is a need for realistic and quantitatively reliable methods to calculate the Auger effects in quantum dots.

We have applied our pseudopotential many-body approach [17] to calculate different Auger processes in CdSe quantum dots. We will show that such calculations produce quantitative agreement with experiments, reveal the dependence of cooling rates on excitation energy, predict the ratios between $\tau_{e-h}^{3 \rightarrow 2}$ and $\tau_{e-h}^{2 \rightarrow 1}$, the hidden relations between $\tau_{e-h}^{2 \rightarrow 1}$ and $\tau_{e}$ and $\tau_{h}$, and the role of the dot surface in Auger multi-exciton recombination.

Method of calculation: although there is no momentum 
conservation in Auger processes for a quantum dot, the energy still needs to be conserved. The discreteness of the dot-confined single-particle energy levels would seem to preclude energy conservation and therefore efficient Auger transitions [13]. However, other interactions having quasi-continuous spectra can be involved and thus mitigate the energy conservation problem. In order to account for these other processes we consider the Auger final states to have a finite lifetime $\hbar / \Gamma$, thus evolve with time as $\Phi_{\text {final }} e p(-i \omega t-\Gamma t / 2 \hbar)$. This finite lifetime is due to interaction with other excitations (e.g., phonons) which cause their decay into lower energy states. We derive a phenomenological formula for the Auger rate (under the standard time dependent perturbation theory):

$$
W_{i}=\frac{\Gamma}{\hbar} \sum_{n} \frac{|<i| \Delta H\left|f_{n}>\right|^{2}}{\left(E_{f_{n}}-E_{i}\right)^{2}+(\Gamma / 2)^{2}},
$$

where $\mid i>$ and $\mid f_{n}>$ are the initial and final Auger electronic states, $E_{f_{n}}$ and $E_{i}$ are their eigen-energies, and $\Delta H$ is the Coulomb interaction. In Eq. (1), we have used multiple final states $\{n\}$ (where $n$ includes spin as well), since each final state might have some contributions to the Auger rate W. The Auger lifetime is $\tau=1 / W_{i}$. We have calculated the single-particle energy levels $\epsilon_{i}$ from the plane-wave empirical pseudopotential method described in Ref. [18], solved within a planewave basis, including spin-orbit effects. The surface of the wurzite dots is saturated by ligand potentials. We have used both the original EPM of ref. [19] (henceforth referred to as EPM-1), and a slightly modified potential (EPM-2), with a different numerical implementation of the non-local potential (the eigenstates of EPM-1 and EPM-2 are however very similar). We consider two dots: $\mathrm{Cd}_{232} \mathrm{Se}_{235}, \mathrm{Cd}_{534} \mathrm{Se}_{527}$, of diameters 29.25 and $38.46 \AA$, respectively. The initial and final states $|i\rangle$ and $|f\rangle$ are given by Slater determinants obtained by populating the appropriate electronic states. When the initial or final states are degenerate or nearly degenerate, a configuration-interaction expansion of the many-body states was used to account for the coupling between the nearly degenerate Slater determinants. The evaluation of the Auger matrix elements $\langle i|\Delta H| f\rangle$ requires the calculation of Coulomb integrals of the form:

$$
\begin{aligned}
J(j, k, l, m)= & \sum_{\sigma, \sigma^{\prime}} \iint \phi_{j}^{*}(\mathbf{r}, \sigma) \phi_{k}^{*}\left(\mathbf{r}^{\prime}, \sigma^{\prime}\right) \frac{e^{2}}{\epsilon\left(\mathbf{r}, \mathbf{r}^{\prime}\right)\left|\mathbf{r}-\mathbf{r}^{\prime}\right|} \\
& \times \phi_{l}(\mathbf{r}, \sigma) \phi_{m}\left(\mathbf{r}^{\prime}, \sigma^{\prime}\right) d^{3} r d^{3} r^{\prime},
\end{aligned}
$$

where $\left\{\phi_{i}\right\}$ are the single-particle wave functions and $\epsilon\left(\mathbf{r}, \mathbf{r}^{\prime}\right)$ is the dielectric function of the quantum dot. Theoretically it is not clear whether the Auger rates should include screening or not (i.e. whether $\epsilon\left(\mathbf{r}, \mathbf{r}^{\prime}\right)=1$ ). Traditionally, in the theoretical treatment of bulk valence Auger processes, dielectric functions are used [20], although there is no rigorous derivation for such screening [21]. It is also important to determine whether the main contribution to the Coulomb integrals (Eq. (2)) comes from the interior of the dot (in which case screening might be important), or from its surface (in which case $\epsilon\left(\mathbf{r}, \mathbf{r}^{\prime}\right) \sim 1$ would be a better approximation). To account for both possibilities, we use a dielectric screening function

$$
\frac{1}{\epsilon\left(\mathbf{r}, \mathbf{r}^{\prime}\right)}=1+\left(\frac{1}{\epsilon\left(d ;\left|\mathbf{r}-\mathbf{r}^{\prime}\right|\right)}-1\right) m(r) m\left(r^{\prime}\right)
$$

where $\mathrm{m}(\mathrm{r})$ is a mask function that changes smoothly from 1, when $r$ is inside the dot, to 0 , when $r$ is outside. $\epsilon\left(\mathbf{r}, \mathbf{r}^{\prime}\right)$, therefore, is equal to $\epsilon\left(d ;\left|\mathbf{r}-\mathbf{r}^{\prime}\right|\right)$ inside the dot, while it is equal to 1 when $r$, or r', or both are outside the dot. Eq. (3) can thus also be used to investigate the origin (surface or interior) of $J(j, k, l, m)$ : if the use of Eq. (3) yields the same result obtained with $\epsilon\left(\mathbf{r}, \mathbf{r}^{\prime}\right)=$ $\epsilon\left(d ;\left|\mathbf{r}-\mathbf{r}^{\prime}\right|\right)$, then the main contribution to the integral comes from the interior of the dot. If, however, the result is close to the one obtained with $\epsilon\left(\mathbf{r}, \mathbf{r}^{\prime}\right) \sim 1$, then the integral is coming mostly from the surface. We have used our calculated dielectric function $\epsilon\left(d ;\left|\mathbf{r}-\mathbf{r}^{\prime}\right|\right)$ [19], which depends on the dot size $d$.

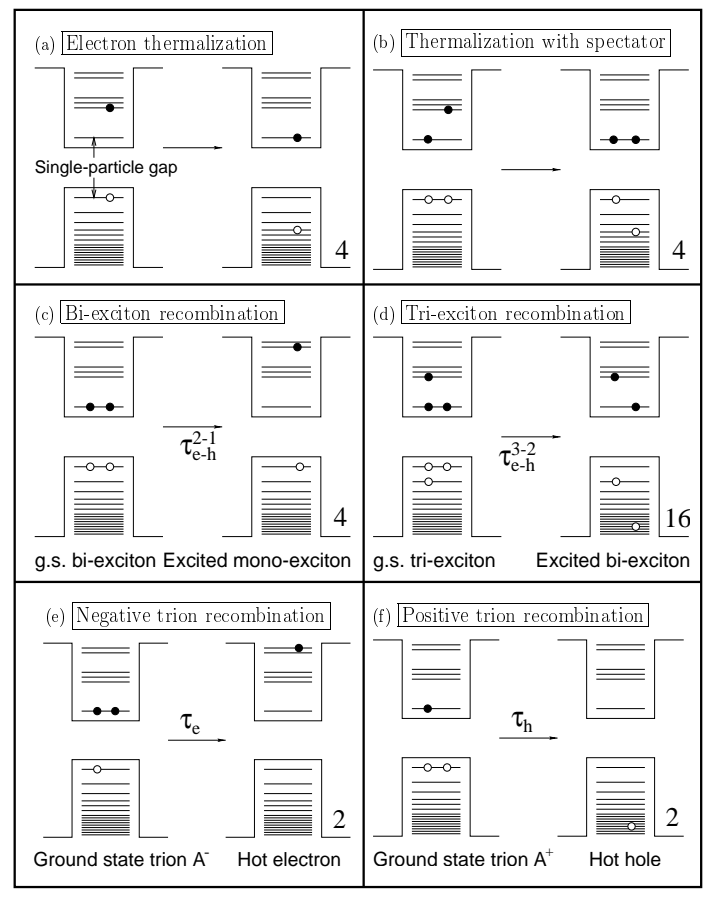

FIG. 1: Illustration of the various Auger decays considered here. The integer on the bottom right-hand side of each panel indicates the total number of spin configurations (channels) in the final state.

Auger electron thermalization: The final and critical step in the electron cooling process (Fig. 1a) involves the decay of the excited electron from the $p$ level $e_{p 1}$ to the ground electronic state $e_{s}$. In the Auger-mediated thermalization process, this is achieved by promoting a 
hole from $h_{s}$ to $h_{n}$. The decay rate is thus:

$\tau^{-1}\left(h_{s} e_{p 1} \rightarrow h_{n} e_{s}\right)=\frac{\Gamma}{\hbar} \sum_{n \alpha} \frac{\left|J\left(h_{s}, e_{p} ; h_{n}, e_{s, \alpha}\right)\right|^{2}}{\left(\Delta E+\epsilon_{h_{n}}-\epsilon_{h_{s}}\right)^{2}+(\Gamma / 2)^{2}}$,

where $\Delta E=\epsilon_{e_{p 1}}-\epsilon_{e_{s}}$ is the energy difference between initial and final electron levels and the sum runs over the $s$ electron spin $\alpha=\uparrow, \downarrow$ as well. Using the masked dielectric function of Eq. (3), we find that the main contribution to the integrals $J\left(h_{s}, e_{p}, h_{n}, e_{s}\right)$ comes from the interior of the dot, so the use of $\epsilon\left(\mathbf{r}, \mathbf{r}^{\prime}\right)=\epsilon\left(d ;\left|\mathbf{r}-\mathbf{r}^{\prime}\right|\right)$ is appropriate for the Auger thermalization process. The summation in Eq. (4) includes 30 final hole states $\left\{\phi_{h_{n}}\right\}$ [22]. The resulting $\tau^{-1}\left(h_{s} e_{p} \rightarrow h_{n} e_{s}\right)$ are plotted as functions of $\Delta E$ in Fig. 2 using three possible values for the broadening $\Gamma$ : 5, 10, $20 \mathrm{meV}$. Experimental energy loss rates for highly excited holes (or electrons) yield an estimate for $\Gamma$ around $10 \mathrm{meV}$ [23]. Since in actual nanocrystals, there are many factors which might affect the value $\Delta E$ of the electron $s p$ splitting (shape and size distribution, surface effects, external charge near the quantum dot, etc.), we show, in Fig. 2 the plot of $\tau$ vs. $\Delta E$, from which we derive the following observations: (i) at resonance, $\tau$ is of the order of $0.1 \mathrm{ps}$; (ii) away from resonance, the Auger lifetime is inversely proportional to $\Gamma$, and, for $\Gamma=10$ $\mathrm{meV}, \tau$ is about $0.5 \mathrm{ps}$ for both quantum dots. These results are in excellent agreement with the recent experiment by Klimov et al. [6], where the $p$ to $s$ electron cooling has been determined to have a lifetime of about $0.3 \mathrm{ps}$.

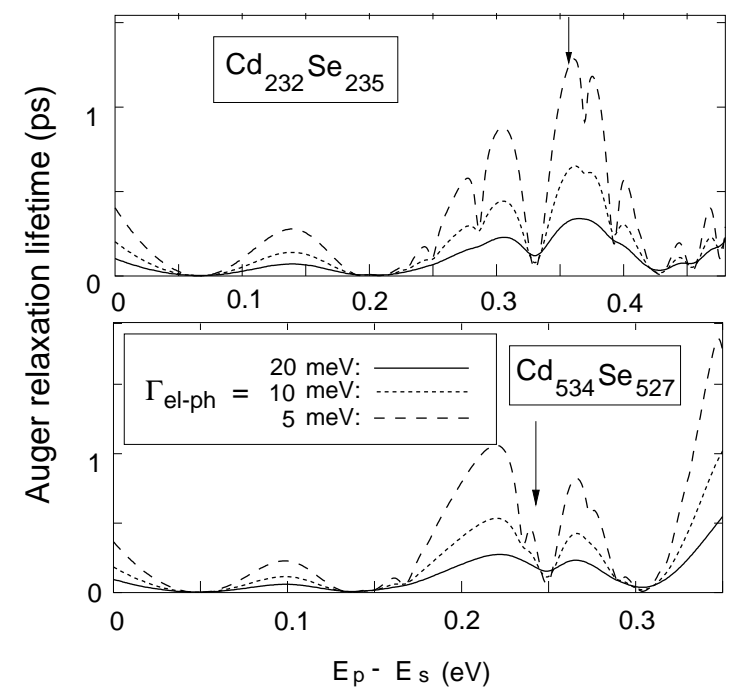

FIG. 2: Auger decay rates for electron cooling (Fig. 1a), for two different sizes of passivated CdSe dots, evaluated at three values of the broadening parameter $\Gamma$ (see Eq. (4)). The vertical arrows denote the value of the cooling energy $\Delta E=\epsilon_{\epsilon_{p}}-\epsilon_{e_{s}}$. The calculations use EPM-2 and the singleparticle approximation.

Many-body effects on Auger thermalization: Table I (left-hand side) compares the results of the single-particle (SP) approach and the CI treatment for $\tau\left(h_{s} e_{p 1} \rightarrow h_{n} e_{s}\right)$ (Fig. 1a), showing that many-body effects play a minor role in such decay. We find that correlation effects underlying the CI treatment lead to a shift of the resonance positions, compared to the single particle treatment. This shift increases with decreasing nanocrystal dimensions, whereas the overall shape of the curves and the values of the lifetimes at resonance are very similar.

TABLE I: Left-hand side: Comparison between electron cooling $e_{p 1} h_{s} \rightarrow e_{s} h_{n}$ Auger lifetimes (in ps) calculated within the single-particle $(S P)$ approximation and with CI. Right-hand side: comparison between SP and CI results for the Auger cooling lifetimes in the presence of a spectator ground state exciton. All values displayed are calculated with EPM-1 (at $\mathrm{T}=300 \mathrm{~K}$ ) for the actual value of the electron $s p$ splitting (i.e. at the position of the arrow in Fig. 2). The CI basis includes the first 30 hole and first 7 electron states, i.e. 840 configurations.

\begin{tabular}{l|cc|cc}
\hline \hline & \multicolumn{2}{|c|}{ No spectator exciton } & \multicolumn{2}{c}{ With spectator exciton } \\
& SP & CI & CI & SP \\
\hline $\mathrm{Cd}_{232} \mathrm{Se}_{235}$ & 0.024 & 0.023 & 0.017 & 0.024 \\
$\mathrm{Cd}_{534} \mathrm{Se}_{527}$ & 0.029 & 0.038 & 0.036 & 0.029 \\
\hline \hline
\end{tabular}

Auger thermalization in the presence of a spectator exciton: thermalization from $e_{p 1}$ to $e_{s}$ can also occur when other particles exist as spectators. We find (Table I righthand side) that the electron cooling lifetime in the presence of a spectator exciton (Fig. 1b) is shorter than the lifetimes for the corresponding Auger relaxation without the spectator exciton [24]. This implies that the correlation effects (partially included in the CI treatment, but not in the single-particle one) become increasingly important, for the Auger electron cooling process, with increasing number of carriers.

Auger bi-exciton recombination (Fig. 1c): The biexciton recombination process sketched in Fig. 1c has an interesting selection rule: if we use $\tau_{e}$ to denote the Auger lifetime for the process of exciton+electron $\rightarrow$ electron (Fig. 1e), and $\tau_{h}$ for the process of exciton+hole $\rightarrow$ hole (Fig. 1f), then we have the rule:

$$
\frac{1}{\tau_{e-h}^{2 \rightarrow 1}}=\frac{2}{\tau_{e}}+\frac{2}{\tau_{h}}
$$

where the factor 2 comes from the increased channel availability in the 2 exciton $\rightarrow 1$ exciton case [25]. To calculate $\tau_{e}$ and $\tau_{h}$, we use a single Slater determinant to represent $\mid i>$ and $\mid f_{n}>$ in Eq. (1), and we obtain: 


$$
\begin{aligned}
\frac{1}{\tau_{e}}= & \frac{1}{\hbar} \sum_{n} \frac{\Gamma}{\left(\epsilon_{g a p}-\epsilon_{e_{n}}+\epsilon_{e_{s}}\right)^{2}+(\Gamma / 2)^{2}} \\
& \times\left|J\left(e_{s, \uparrow}, e_{s, \downarrow} ; e_{n}, h_{s}\right)-J\left(e_{s, \downarrow}, e_{s, \uparrow} ; e_{n}, h_{s}\right)\right|^{2},
\end{aligned}
$$

and

$$
\begin{aligned}
\frac{1}{\tau_{h}}= & \frac{1}{\hbar} \sum_{n} \frac{\Gamma}{\left(\epsilon_{g a p}+\epsilon_{h_{n}}-\epsilon_{h_{s}}\right)^{2}+(\Gamma / 2)^{2}} \\
& \times\left|J\left(h_{s, \uparrow}, h_{s, \downarrow} ; h_{n}, e_{s}\right)-J\left(h_{s, \downarrow}, h_{s, \uparrow} ; h_{n}, e_{s}\right)\right|^{2},
\end{aligned}
$$

where the subscripts $\uparrow, \downarrow$ indicate the spin- degenerate Kramer's doublets, and $\epsilon_{\text {gap }}$ is the single-particle energy gap (see Fig. 1a). Via Eq. (3), we find that the multiexciton recombination rate comes primarily from the surface of the dot, so the screening $\epsilon\left(\mathbf{r}, \mathbf{r}^{\prime}\right)$ can be assumed in first approximation to be equal to 1 . To calculate $\tau_{e}$ $\left(\tau_{h}\right)$ we have computed 60 electron (hole) states around the ideal energy $\epsilon_{g a p}+\epsilon_{e_{s}}\left(\epsilon_{g a p}-\epsilon_{h_{s}}\right)$. The results for $C d_{534} S e_{527}$ are shown in Fig. $3 \mathrm{a}$, for $\Gamma=10 \mathrm{meV}$. The lifetime $\tau$ is plotted as a function of $\epsilon_{g a p}$, the actual value of which is indicated by a vertical arrow. We see how the sum rule (Eq. (5)) is obeyed: The slow decay of the negative trion into a hot electron $\left(\tau_{e} \sim 40-60 \mathrm{ps}\right.$, in Fig. $1 \mathrm{e})$, and the decay of the positive trion into a hot hole ( $\tau_{h} \sim 40-80$ ps, in Fig. 1f), add up to the fast bi-exciton decay $\tau_{e-h}^{2 \rightarrow 1}$, Fig. 1c, of about $12 \mathrm{ps}$. We estimate that the use of the effective screening of Eq. (3) will increase the calculated Auger lifetime by a factor of about 2, yielding $\tau_{e-h}^{2 \rightarrow 1} \sim 24$ ps. This is in excellent agreement with the experimental result of $22 \mathrm{ps}$ [9].

Tri-exciton Auger decay (Fig. 1d): The values obtained for the lifetime as a function of the single-particle gap $\epsilon_{\text {gap }}$, assuming $\epsilon\left(\mathbf{r}, \mathbf{r}^{\prime}\right)=1$, are shown in Fig. 3b for $C d_{534} S e_{527}$. We see that, $\tau_{e-h}^{3 \rightarrow 2}$ is roughly 5 ps. This gives a ratio of $\tau_{e-h}^{2 \rightarrow 1} / \tau_{e-h}^{3 \rightarrow 2}=2.4$, which is very close to the experimental ratio of 2.1 [9].

In summary, we find: (i) A sensitive dependence of the electron cooling rates on $E_{p}-E_{s}$, the off-resonance value of which depends on the value of $\Gamma$. Due to this sensitivity it should not be possible experimentally to see single exponential decay when investigating dot ensembles. Using the experimentally estimated $\Gamma(10 \mathrm{meV})$, gives $\tau$ of the order of $0.5 \mathrm{ps}$, in excellent agreement with experiment. (ii) The main contribution to $1 / \tau_{e-h}^{2 \rightarrow 1}$ comes from the dot surface, therefore its exact value might depend on the details of the description of the surface dielectric screening. (iii) A simple model for the screening function near the surface gives an estimate for $\tau_{e-h}^{2 \rightarrow 1}$ around $24 \mathrm{ps}$ for the $\mathrm{Cd}_{534} \mathrm{Se}_{525}$ dot. This again is in very good agreement with experiments. (iv) Our calculated ratio $\tau_{e-h}^{2 \rightarrow 1} / \tau_{e-h}^{3 \rightarrow 2}=2.4$ for the $C d_{534} S e_{527}$ dot agrees well with the experimental value of 2.1 .

Our pseudopotential calculations confirm many experimental Auger results in CdSe quantum dots. Our

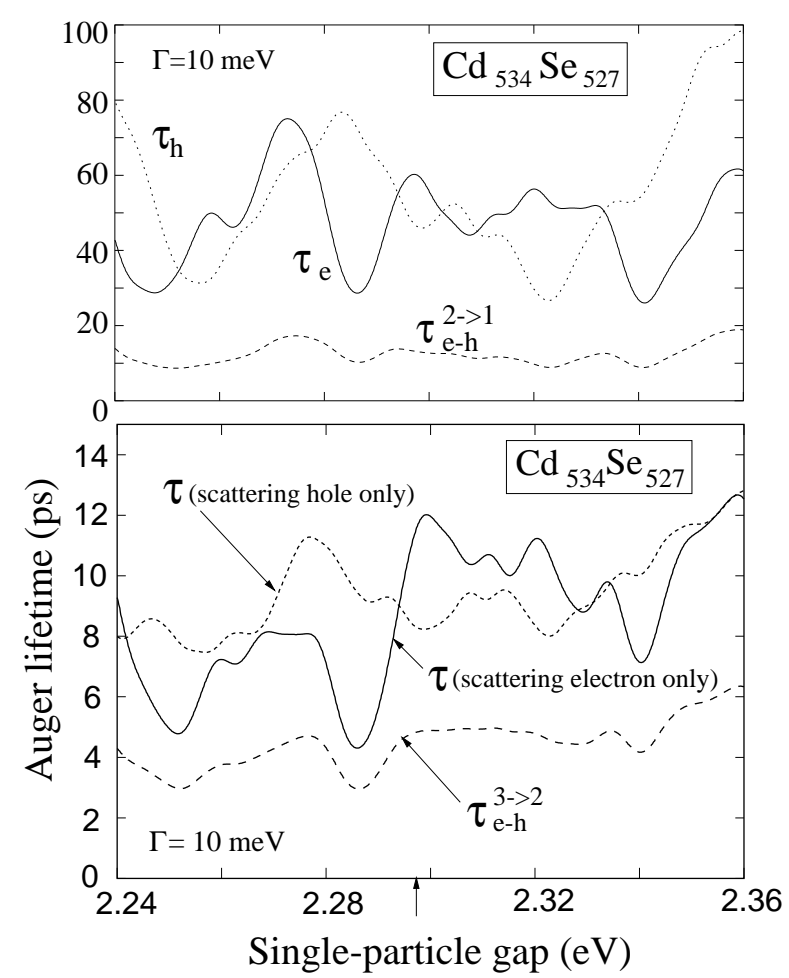

FIG. 3: $C d_{534} S_{527}$ dot: (a) Auger bi-exciton recombination lifetime $\tau_{e-h}^{2 \rightarrow 1}$ (Fig. 1b), decomposed into the $\tau_{e}$ (Fig. 1c) and $\tau_{h}$ (Fig. 1d) contributions (see Eq. (5)). (b) Auger tri-exciton recombination lifetime $\tau_{e-h}^{3 \rightarrow 2}$ together with its two contributions coming from a single hole and a single electron scattering. All lifetimes were calculated with EPM-2 and $\epsilon\left(\mathbf{r}, \mathbf{r}^{\prime}\right)=1$.

methodology presents itself as a reliable tool to calculate the details of Auger processes in nanostructures.

The work at NERSC was supported by U.S. DOE, OER-BES, under Grant No. KC0203010, at NREL by the U.S. DOE, OER-BES, Division of Materials Science.

[1] V. I. Klimov, J. Phys. Chem. B 104, 6112 (2000).

[2] H. Benisty, C. M. Sotomayor-Torres, and C. Weisbuch, Phys. Rev. B 44, 10945 (1991).

[3] T. Inoshita and H. Sakaki, Phys. Rev. B 46, 7260 (1992).

[4] X.-Q. Li, H. Nakayama, and Y. Arakawa, Phys. Rev. B 59, 5059 (1999).

[5] S. Sauvage et al., Phys. Rev. Lett. 88, 177402 (2002).

[6] V. I. Klimov and D. W. McBranch, Phys. Rev. Lett. 80, 4028 (1998).

[7] P. Guyot-Sionnest, M. Shim, C. Matranga, and M. Hines, Phys. Rev. B 60, R2181 (1999).

[8] Al. L. Efros, V. A. Kharchenko, and M. Rosen, Solid State Comm. 93, 281 (1995).

[9] V. I. Klimov et al., Science 287, 1011 (2000).

[10] M. Nirmal et al., Nature 383, 802 (1996). 
[11] J. H. Werner, S. Kolodinski, H. J. Queisser, Phys. Rev. Lett. 72, 3851 (1994).

[12] V. I. Klimov and D. McBranch, Phys. Rev. B 55, 13173 (1997).

[13] J. L. Pan, Phys. Rev. B 49, 11272 (1994); J. L. Pan, Phys. Rev. B 46, 3977 (1992).

[14] C. Delerue, G. Allan, and M. Lannoo, Phys. Rev. B 64, 193402 (2001); C. Delerue et al., Phys. Rev. Lett. 75, 2228 (1995).

[15] L.-W. Wang and A. Zunger, J. Phys. Chem B 102, 6449 (1998).

[16] L.-W. Wang, Phys. Rev. B 61, 10235 (2000).

[17] A. Zunger, Phys. Stat. Solidi B 224, 727 (2001).

[18] L.-W. Wang and A. Zunger, Phys. Rev. B 51, 17398 (1995); H. Fu and A. Zunger, Phys. Rev. B 56, 1496 (1997).
[19] A. Franceschetti, H. Fu, L.-W. Wang and A. Zunger, Phys. Rev. B 60, 1819 (1999).

[20] M. Takeshima, J. Appl. Phys. 43, 414 (1972).

[21] A. Haug and W. Ekardt, Solid State Comm. 17, 267 (1975).

[22] We tested the choice of the Auger basis by calculating $\tau\left(h_{s} e_{p 1} \rightarrow h_{n} e_{s}\right)$ with 12 hole states with energy around $\epsilon_{h_{s}}-\Delta E$ and with the first 30 hole states, finding differences of less than $2 \%$, within the single-particle approach.

[23] D. J. Norris, M. Nirmal, A. Sacra, C. B. Murray, and M. G. Bawendi, Z. Phys. D 26, 355 (1993).

[24] In the single-particle treatment there is no difference between the Auger electron cooling lifetimes calculated with and without spectator exciton.

[25] We neglect correlation effects in Eq. (4). 